\title{
ACEsso universal aO Sistema DE TeleFonia FiXa nO BRasIL: ESQUEMAS ALTERNATIVOS DE FNANCIAMENTO E AVALIAÇÃO DOS IMPACTOS DISTRIBUTIVOS SOBRE O BEM-ESTAR DAS FAMILIAS
}

\author{
Paulo Amilton Maia Leite Filho \\ Professor do Departamento de Economia da UFPB. \\ Doutor em Economia pela \\ Universidade Federal de Pernambuco - PIMES. \\ pmaiaf@ripmail.com.br
}

\section{RESUMO}

Atualmente a infra-estrutura de informação adquire cada vez mais importância, dado que a partir dela é possível acelerar as transformações sociais e econômicas. Esta infra-estrutura tem como um de seus insumos fundamentais o acesso ao sistema de telecomunicação, notadamente o sistema de telefonia fixa. $\mathrm{O}$ aumento do número de usuários conectados aquele sistema é essencial para que a infra-estrutura de informação possa reverter à sociedade todos os seus benefícios potenciais. Em Leite Filho (2000) é mostrado que existe um impacto positivo para a sociedade de universalizar o acesso à telefonia fixa no Brasil.

Neste sentido, o estudo aqui exposto tem como propósito avaliar se para as famílias que compõe a sociedade brasileira aquele resultado se mantém. A metodologia consistiu na utilização da variação equivalente. $\mathrm{O}$ resultado deste estudo mostra que os impactos da universalização para as famílias é positivo.

Palavras Chaves: Externalidades, Universalização do acesso a Telefonia Fixa, Bem-estar das Famílias, Variação Equivalente e Bens de Rede.
$\operatorname{ABSTR} A C T$

Currently the information infrastructure acquires each time more importance, given that it is seen as a trigger to speed up social and economic transformation. This infrastructure has as the one of its basic inputs the access to the telecommunication system, mainly the system of fixed telephony. The increase in the number users connected to that system is essential to allow the information infrastructure to revert to the society all its potential benefits. In Leite Filho (2000), it is shown that universalization of access of the fixed telephony in Braqil causes a positive impact on the society. In this direction, this study intends to evaluate if for the families that make up the Braqilian society such result is maintained. The methodology employed was equivalent variation. The results show that the impact of universalization on the families is positive.

Key Words: Externalities, Universalization of the access the Fixed Telephony, Families Welfare, Equivalent Variation and Wide Goods. 


\section{INTRODUÇÃO}

Nas sociedades contemporâneas a infra-estrutura de informação adquire cada vez mais importância, dado que a partir dela a sociedade tem possibilidade de obter benefícios sociais e econômicos cada vez mais acentuados com o aumento da velocidade do processo informativo.

Os benefícios sociais são obtidos, principalmente, pelos avanços nas áreas de educação e saúde, enquanto os econômicos resultam da possibilidade de tornar os fatores de produção mais eficientes, na ampliação da competição nos mercados, na melhoria da eficácia governamental etc. Todos esses benefícios contribuem potencialmente para a elevação do bemestar das famílias.

A infra-estrutura de informação tem como um de seus insumos fundamentais o acesso ao sistema de telecomunicação, notadamente o sistema de telefonia fixa. Quanto maior for o número de usuários que tem acesso a esse sistema, maiores serão as possibilidades daqueles benefícios acontecerem devido as externalidades de rede provenientes do aumento da quantidade de novos usuários conectados ao sistema.

Segundo Economides (1996) e (1994), a telefonia se distingue de muitos outros bens pelo fato de sua utilização não envolver apenas um usuário, mas sim uma rede de no mínimo dois usuários. Desta forma, telefonia pode ser definida como um bem de rede de dois estágios, dado que seu consumo tem como objetivo final conectar duas partes distintas.

Uma característica fundamental associada aos bens de rede, e que esteve na base da argumentação de Leite Filho (2000) para justificar que tais bens geram ganhos de bem-estar para a sociedade brasileira, é a existência de externalidade ${ }^{1}$ de rede positiva. A adição de um novo usuário, numa rede de $n$ ramificações já existente, cria 2 n novos bens compostos potenciais. A externalidade de rede envolve a criação de novos bens para os antigos usuários, já que, com a mesma quantidade de insumo, pode-se multiplicar a quantidade de bens. Isto significa que o valor do sistema telefônico aumenta com o número de unidades que podem ser acessadas ${ }^{2}$.

No caso do sistema de telefonia fixa, a externalidade de rede existe pelo fato de permitir comunicação com um número maior de pessoas e, por conseqüência, maior contato interpessoal, possibilitando maiores ocorrências de trocas de informações entre os agentes.

Consciente da possibilidade de criação de enormes externalidades de rede, a Agência Nacional das Telecomunicações (ANATEL), com base na Lei Geral das Telecomunicações n. ${ }^{\circ}$ 9.472, estabeleceu que o sistema de telefonia fixa deveria ser expandido em 13 milhões de novas linhas, até o ano de $2001^{3}$. Leite Filho (2000) mostrou que aquele aumento do número de usuários ao sistema de telecomunicação fixo apresentaria impactos positivos para a sociedade brasileira como um todo, sem dividi-la em famílias.

A título de comparação com as metas estabelecidas pela ANATEL, aquele estudo elegeu um quantitativo de novos acessos alternativos, que teve a renda como indicador sócio-econômico comum. A justificativa da escolha deste atributo é que ele exibia uma distribuição desigual entre aquelas famílias que têm ou não acesso ao sistema telefônico no Brasil, como mostra a tabela 1. A renda média mensal das famílias que não tinham acesso em 1996 ao sistema telefônico ficava na faixa de $\mathrm{R} \$ 510,27$, enquanto que aquelas que tinham acesso possuem renda média mensal de $\mathrm{R} \$ 2.025,34$, ou seja, quatro vezes maior. Desta for$\mathrm{ma}$, as famílias que dispunham de renda média em torno daquele valor formaram o quantitativo alternativo de novos acessos em Leite Filho (2000). Segundo a Pesquisa Nacional de Amostragem por Domicílio (PNAD) o número de famílias que tinha em 1996 renda em torno de $\mathrm{R} \$ 510,27$ era de 6.518.167.

A universalização do acesso à telefonia fixa deveria ser viabilizada por um fundo, como estabelece o artigo 81 da Lei Geral das Telecomunicações de n. ${ }^{\circ}$ 9.472. Este tinha a função de prover o financiamento àquelas metas de expansão. Este fundo se mostrou um instrumento necessário, em primeiro lugar, por que grande parte da população brasileira não tem renda suficiente para adquirir o acesso ao sistema de telecomunicação fixo e, em segundo lugar, pelas suas hipotéticas externalidades positivas à sociedade advindas do aumento de novos usuários daquele sistema.

$\mathrm{O}$ artigo 81 da referida lei estabeleceu três maneiras alternativas para a constituição daquele fundo. Foram elas:

-Transferências do Orçamento Geral da União, dos Estados, do Distrito Federal e dos Municípios;

- Subsídios entre modalidades de serviços ou segmentos de usuários; $\mathrm{e}$

- Pagamento de um adicional ao valor das interconexões entre as operadoras do sistema.

Leite Filho (2000) adotou a primeira destas alternativas como forma de criar o fundo para viabilizar a universalização do acesso à telefonia fixa no Brasil. Dentre esta alternativa foram utilizados os impostos indiretos. A justificativa desta escolha foi o fato de que os impostos indiretos incidem em todos os estágios da produção e

\footnotetext{
1 Pode se definir externalidades com uma cesta de bens que um usuário oferece a qualquer outro sem que estes estejam realizando qualquer tipo de transação.

2 Ver Economides (1996).

3 Essa meta teve como base a Lei Geral das Telecomunicações n. ${ }^{\circ}$ 9.472, de 16 de junho de 1997, em seu artigo 63, que específica a política de universalização do acesso ao sistema de telefonia fixa no Brasil.
} 
comercialização, sendo possível aumentar o universo de contribuintes. Desta feita, aquele trabalho teve como objetivo avaliar os possíveis impactos das modificações nos impostos indiretos necessárias para viabilizar a política de universalização do acesso ao sistema de telefonia fixa do governo brasileiro sobre o bem-estar da sociedade.

Tabela 1- Brasil - Rendimento Mínimo, Médio e Máximo Domiciliar Segundo a Condição de Propriedade da Linha Telefônica (R\$/Mês)

\begin{tabular}{lcc}
\hline Rendimento/ Tem telefone? & Sim & Não \\
\hline Mínimo & 0 & 0 \\
Médio & $2.025,34$ & 510,27 \\
Máximo & $63.500,00$ & $35.100,00$ \\
\hline
\end{tabular}

Fonte : IBGE - PNAD- 96. Obs. Valores estão em reais de setembro de 1996.

\section{BASE TEÓRICA}

As modificações nas alíquotas dos impostos indiretos, necessárias para estabelecer o fundo de financiamento do acesso universal ao sistema de telecomunicação, têm efeitos sobre o consumo das famílias. Portanto, é necessário se comparar os níveis de bem-estar quando as famílias enfrentam diferentes conjuntos de possibilidades de consumo, evidenciando a conexão entre a análise de bem-estar das famílias e a teoria da demanda. A relação entre a mensuração do bem-estar e a teoria da demanda parte do conceito de função utilidade indireta, expressa da seguinte forma:
Os resultados de Leite Filho (2000) foram que o impacto do aumento na quantidade de novos usuários no bem-estar da sociedade brasileira era positivo, tanto para o quantitativo estabelecido pela ANATEL como para o alternativo estabelecido com base na renda dos não usuários. Ou seja, o benefício de ter o acesso ao sistema de telefonia fixa é maior do que os custos de viabilizá-lo. Naquele contexto, o aumento dos impostos indiretos foram compensados pelo benefício do acesso, segundo Leite Filho (2000).

No entanto, a sociedade brasileira é composta de famílias com perfis de renda/gastos distintos e que podem exibir impactos diferenciados no seu bem-estar quando os impostos indiretos sofrem elevação para viabilizar o acesso à telefonia fixa. Ou seja, para algumas famílias o benefício do acesso à telefonia fixa pode ser suplantado pelo custo representado pelo aumento dos impostos indiretos, mesmo que para a sociedade os benefícios suplantem os custos. Sendo assim, o presente estudo tem como objetivo avaliar os impactos sobre o bem-estar das famílias brasileiras do aumento do número de usuários do sistema de telefonia fixa. A quantidade de novos acessos serão aqueles mesmos de Leite Filho (2000), bem como as modificações nas alíquotas dos impostos indiretos. O estudo é dividido da seguinte forma, além da introdução, temos a base teórica na seção 2, o modelo matemático na seção 3 e os resultados na seção 4. Por fim a bibliografia.

$$
\mathrm{V}\left(\mathrm{q}, \mathrm{y}^{\mathrm{j}}\right)=\max \mathrm{u}^{\mathrm{j}}\left(\mathrm{x}^{\mathrm{j}}\right) \text { sujeito }{ }_{j=1}^{\mathrm{a}} \sum_{\mathrm{j}=1}^{\mathrm{j}} \leq \mathrm{y}^{\mathrm{j}}
$$

Onde $\mathrm{j}$ indica as famílias, q o vetor de preços já incluído os impostos indiretos, $\mathrm{y}^{\mathrm{j}}$ a renda, $\mathrm{x}^{\mathrm{j}}$ o vetor de quantidades demandadas e u' a função utilidade, todos da família j. $V\left(q, y^{\prime}\right)$ fornece a utilidade máxima, dados os preços e a renda de j. Equivalentemente, $V\left(q, y^{j}\right)$ expressa a cesta que contém as quantidades demandadas máximas de bens que uma família pode adquirir, dados os preços dos bens e a renda da mesma.

Assumindo que a ordenação das preferências satisfaz os axiomas da racionalidade ${ }^{4}$, continuidade, convexidade e não saciedade local ${ }^{5}$, então $\mathrm{V}\left(\mathrm{q}, \mathrm{y}^{\mathrm{j}}\right)$ apresenta algumas propriedades que permitem invertê-la ${ }^{6}$ de tal forma que $y^{j}$ seja função de um nível específico de utilidade. Isto é, para cada nível de utilidade constante $\mathrm{u}_{\mathrm{c}}^{\mathrm{j}}$ existe um montante mínimo de $y^{j}$ necessário para se obter aquele nível de utilidade, aos preços q. A função que relaciona o nível de gasto e a utilidade constante é conhecida como função gasto, e ( $\left.q, u_{c}^{j}\right)$, e tem sua definição dada por:

$$
\mathrm{e}\left(\mathrm{q}, \mathrm{u}_{\mathrm{c}}^{\mathrm{j}}\right)=\min _{\mathrm{j}=1}^{\mathrm{n}} \sum_{\mathrm{q}} \mathrm{x}^{\mathrm{j}} \text { sujeito } a \mathrm{u}_{\mathrm{c}}^{\mathrm{j}}
$$

4 Uma ordenação racional implica que ela é reflexiva, comparável e transitiva. Ver Mas-Colell et al. (1995), Varian (1992) e Kreps (1990).

5 A racionalidade implica que as preferências são reflexivas, completas e transitivas. A convexidade implica que consumidor prefere consumir combinações de bens. Não saciedade local implica que os consumidores sempre irão preferir cestas que contenham quantidades maiores de bens do que quaisquer outras.

6 Ver Mas-Colell et al. (1995), Varian (1992) e Kreps (1990). 
A expressão (2) mostra o gasto mínimo necessário para se obter à utilidade $u_{c}^{j}$ desejada. A dualidade ${ }^{1}$ mostra que $V(q$, $\left.y^{j}\right)$ e e $\left(q, u_{c}^{i}\right)$ geram o mesmo resultado, ou seja, a cesta de bens que maximiza a utilidade é a mesma que minimiza os gastos. Desta feita, pode-se utilizar e $\left(\mathrm{q}, \mathrm{u}_{\mathrm{c}}^{\mathrm{j}}\right)$ para se encontrar medidas de variação de bem-estar.

Fazendo uma transformação monotônica de e $\left(\mathrm{q}, \mathrm{u}_{\mathrm{c}}^{\mathrm{i}}\right)^{2}$, podemos encontrar a função utilidade de métrica monetária $\mathrm{m}\left(\mathrm{q}^{\mathrm{r}}, \mathrm{q}, \mathrm{y}^{\mathrm{i}}\right)$, que mede o quanto de renda uma família necessitaria aos preços de referência $\mathrm{q}^{\mathrm{r}}$ para estar tão bem quanto estava aos preços q com renda $y^{j}$.

Esta função pode ser utilizada como um indicador do padrão de vida das famílias, ao permitir a mensuração da variação de bem-estar associada às mudanças de preços. Para uma família com renda $\mathrm{y}^{\dagger}$, dada uma mudança de preço de $\mathrm{q}^{0}$ para $\mathrm{q}^{1}$, o impacto sobre o seu bem-estar pode ser medido pela diferença nas utilidades em decorrência da variação de preço, expressa por:

$$
\mu\left(q^{r}, q^{1}, y^{j}\right)-\mu\left(q^{r}, q^{0}, y^{j}\right)
$$

Usualmente, adota-se $\mathrm{q}^{0}$ ou $\mathrm{q}^{1}$ como preço de referência $\left(\mathrm{q}^{\mathrm{r}}\right)$. Se utilizarmos $\mathrm{q}^{0}$ como preço de referência, obteremos a medida de variação equivalente (VE'). Esta mede o quanto de recursos monetários teriam que ser dados (ou retirados) às (das) famílias, antes da mudança de preço, para que elas obtenham o mesmo nível de utilidade que teriam depois da variação de preço. A VE j $^{i}$ pode também ser escrita da seguinte forma:

$$
V E^{j}=\mu\left(q^{0}, q^{1}, y^{j}\right)-\mu\left(q^{0}, q^{0}, y^{j}\right)=\mu\left(q^{0}, q^{1}, y^{j}\right)-y^{j}
$$

Se utilizarmos $\mathrm{q}^{1}$ como preço de referência, obteremos a medida de variação compensatória $\left(\mathrm{VC}^{\mathrm{j}}\right)$, que mede a renda necessária para compensar as famílias pela mudança de preço. Isto é, $\mathrm{VC}^{j}$ representa o montante de recursos monetários que devem ser dados (ou retirados) às (das) famílias, depois da modificação no preço, para que elas se mantenham no mesmo nível de utilidade que se encontravam antes da mudança verificada no preço. Sua formulação matemática é a seguinte:

$$
V C^{j}=\mu\left(q^{1}, q^{1}, y^{j}\right)-\mu\left(q^{1}, q^{0}, y^{j}\right)=y^{j}-\mu\left(q^{1}, q^{0}, y^{j}\right)
$$

No entanto, como vamos comparar mudanças tributárias alternativas, temos que utilizar um vetor de preços comum. Neste caso, utilizaremos $\mathrm{q}^{0}$ como preço comum e a $\mathrm{VE}^{j}$ como medida de variação no bem-estar das famílias. Se a VE for positiva, o benefício pela universalização do acesso à telefonia fixa para a família j é maior do que custo de financiá-la. Se a VE' for negativa, o contrário acontece.

\section{MODELO MATEMÁTICO}

Esta seção descreve o modelo matemático que tem como objetivo a simulação dos impactos sobre o bem-estar das famílias provenientes das modificações no sistema tributário indireto. Tais modificações tiveram o intuito de arrecadar o montante de recursos monetários para financiar a universalização do acesso ao sistema de telefonia fixa. Em particular, o modelo é construído no sentido de mostrar a situação em que o governo deseja tributar as mercadorias para amealhar recursos tanto para manter o nível de arrecadação como para financiar o acesso universal à telefonia fixa.

Assume-se que as modificações no sistema tributário podem ser completamente caracterizadas em termos dos efeitos sobre as rendas das famílias e os preços que as famílias enfrentam. Isto implica dizer que os preços dos produtores permanecem constantes. Além do mais, os preços pagos pelas famílias foram considerados independentes das ações destas.

O modelo distingue entre famílias residentes em regiões metropolitanas daquelas que residem em regiões não metropolitanas. Essas duas categorias de famílias foram divididas, por sua vez, em dez grupos, de acordo com seus recebimentos mensais. Todas as famílias em um dado grupo de recebimento mensal foram consideradas de comportamento idêntico, de modo que a maneira de agir do grupo possa ser descrito por uma família que a represente. Assume-se que a renda das famílias das regiões não metropolitanas é metade da renda das famílias das regiões metropolitanas. Assume-se ainda que:

1) A renda familiar é dada;

2) A análise admite que não existe poupança, de tal maneira que o consumidor gasta toda sua renda no consumo dos bens;

3) As famílias recebem do governo uma transferência lump sum ${ }^{9}$, igual a I, que é o montante de recursos monetários necessários para que elas tenham acesso ao sistema de telefonia fixa.

Como não se teve disponível informação suficiente para que se mensure diretamente os benefícios recebidos pelas famílias com o acesso à telefonia fixa, admite-se que I fosse uma "proxy" desses benefícios. Sendo assim, aqueles benefícios são constantes para todas as famílias.

Ao se adotar a hipótese acima, o estudo restringiu o real valor dos benefícios que as famílias obtêm pelo fato de disporem de acesso à telefonia fixa. $\mathrm{O}$ valor daquele benefício depende da composição das famílias bem como das relações que estas mantém com as demais que compõem a sociedade. Para obtermos o valor exato dos benefícios eram necessários dados muito desagregados. No entanto, tal informação não estava disponível no momento da realização deste trabalho.

7 Ver Mas-Colell et al. (1995), Varian (1992) e Kreps (1990).

8 Uma transformação monotônica é aquela que não altera a ordenação das preferências contidas em e(q, ujc). Para maiores detalhes ver Mas-

Colell et al. (1995), Varian (1992) e Kreps (1990).

9 Para maior discussão sobre o emprego desta hipótese ver Graham, Cornford, e Marvin (1998), Taylor (1994) e King (1983). 
4) O custo para as famílias, como resultado da implementação da política de universalização do acesso ao sistema de telefonia fixa, foi representado pelas modificações ocorridas nos preços dos bens que as famílias enfren$\operatorname{taram}\left(\mathrm{q}_{\mathrm{i}}\right)$ em razão das alterações nos impostos indiretos.

A restrição orçamentária por parte das famílias é dada pela seguinte equação:

$$
\sum_{i=1}^{n} q_{i} x_{i}^{j}=y^{j}+I=Y^{j}
$$

Onde: $\mathrm{Y}^{\mathrm{i}}=$ renda total recebida pela família $j ; \mathrm{x}_{i}^{j}=$ consumo da família j pelo bem $\mathrm{i} ; \mathrm{y}^{j}=$ renda da família j; $\mathrm{I}=$ transferência lump sum; e $_{\mathrm{i}}=$ preço do bem $\mathrm{i}$ que a família j enfrenta.

Os preços $\mathrm{q}_{\mathrm{i}}$ foram obtidos ao se acrescentar aos preços dos produtores $\left(\mathrm{p}_{\mathrm{i}}\right)$ os impostos indiretos $\left(\mathrm{t}_{\mathrm{i}}\right)$, como mostra a identidade (4).

$$
\mathrm{q}_{\mathrm{i}}=\mathrm{p}_{\mathrm{i}}+\mathrm{t}_{\mathrm{i}}
$$

Os impostos indiretos foram obtidos da seguinte forma:

$$
\mathrm{t}_{\mathrm{i}}=\theta_{\mathrm{i}} /\left(1-\theta_{\mathrm{i}}\right)
$$

Onde $\mathrm{q}_{\mathrm{i}}$ representa as alíquotas efetivas dos impostos indiretos que recaem sobre bem i. Desta maneira, admitindo que $\mathrm{p}_{\mathrm{i}}$ é igual a 1 , temos que $\mathrm{q}_{\mathrm{i}}$ é dado pela expressão:

$$
\mathrm{q}_{\mathrm{i}}=1 /(1-\theta)
$$

O valor da transferência lump sum (I) foi de $\mathrm{R} \$ 50,00$. Esta quantia representa o custo médio do acesso a uma linha telefônica fixa que era cobrado das famílias, em setembro de 1996, pelas operadoras do sistema de telecomunicação fixo e que foi admitido por Leite Filho (2000).

Admitiu-se que as operadoras do sistema assumiram os custos de instalação que envolveu, entre outros, os gastos com cabeamento da rede, a implantação das centrais comutadoras etc. Esta hipótese se justifica pelo fato de que se quer isolar os efeitos do acesso à rede de teleco- municação, ou seja, em relação ao preço de manter conectado à rede e não ao custo da conexão física inicial.

Como em Leite Filho (2000), dois grupos diferentes de famílias tiveram direito ao acesso à telefonia fixa. O primeiro grupo, denominado de A, foi estabelecido pela Agência Nacional das Telecomunicações (ANATEL) e tem por base o número de famílias que aquela agência estabeleceu atender para os anos de 1999 à 2001 e que constaram no plano de metas daquela agência.

O segundo grupo, denominado de $\mathrm{B}$, tem como base o número de famílias com renda média de $\mathrm{R} \$ 510,27$, também para setembro de 1996. Foi realizada uma comparação dos impactos no bem-estar das famílias e no bem-estar social para esses dois grupos. A quantidade de famílias em ambos os grupos constam da tabela 2 .

Tabela 2 - Brasil - Número de Unidades Familiares do Grupo A e B e seus Respectivos Custos

\begin{tabular}{ccc}
\hline Grupo & Número de Unidades Familiares & $\mathbf{F}_{\mathbf{g}}(\mathbf{R} \mathbf{)}$ \\
\hline $\mathrm{A}$ & 13.000 .000 & 650.000 .000 \\
$\mathrm{~B}$ & 6.518 .167 & 325.908 .356
\end{tabular}

Fonte: Plano de Metas da ANATEL e IBGE, PNAD-1996.

Da equação (6) pode-se conseguir os preços dos consumidores $\left(\mathrm{q}_{\mathrm{i}}\right)$, desde que tenhamos as alíquotas efetivas dos impostos indiretos. Estas foram obtidas de Siqueira et al. (1998), Siqueira (1997) e Siqueira (1995) e são mostradas na tabela 3. Para efeito de simplificação, os impostos indiretos foram classificados em apenas duas categorias, impostos sobre circulação de mercadorias e serviços (ICMS) e outros. O motivo desta agregação é o fato do ICMS ser atualmente o principal imposto indireto no Brasil.

Tabela 3 - Brasil - Alíquotas Efetivas dos Impostos Indiretos (\%)

\begin{tabular}{lccc}
\hline Bens & Aliquota ICMS & $\begin{array}{c}\text { Alíquota Outros } \\
\text { Impostos Indiretos }\end{array}$ & $\begin{array}{c}\text { Alíquota Todos } \\
\text { Impostos Indiretos }\end{array}$ \\
\hline Alimentação & 13,023 & 5,380 & 18,403 \\
Habitação & 8,195 & 3,979 & 12,174 \\
Telefone & 27,752 & 1.602 & 29,354 \\
Vestuário & 18,593 & 3,118 & 21,711 \\
Transporte & 13,960 & 8,836 & 22,796 \\
Cuidados Pessoais & 5,537 & 5,055 & 10,592 \\
Outros & 9,664 & 9,664 & 29,560 \\
\hline
\end{tabular}

Fonte : Siqueira et Al (1998), Siqueira (1997) e Siqueira (1995). 
Desta forma, os preços que os consumidores pagaram, para o caso base, foram calculados pela equação (6) e são os seguintes:

Tabela 4 - Preços dos Consumidores $\left(q_{i}\right)$ Para o Caso Base

\begin{tabular}{lc}
\hline Categoria de Bens & Caso Base \\
\hline Alimentação & 1,22553 \\
Habitação & 1,13861 \\
Telefone & 1,41550 \\
Vestuário & 1,27731 \\
Transporte & 1,29526 \\
Cuidados Pessoais & 1,11846 \\
Outros & 1,41964 \\
\hline
\end{tabular}

Fonte : Cálculo realizado pelo autor utilizando a equação 6 . ram-se os novos preços dos bens, tanto para o grupo A como para o B de famílias, utilizando novamente a equação (6). Os novos valores são apresentados nas tabelas 5 e 6 abaixo.

Os dados utilizados neste estudo, como em Leite Filho (2000), e que representam a demandas das famílias, foram retirados da Pesquisa de Orçamentos Familiares (POF), para o período 1995-96, realizada pelo Instituto Brasileiro de Geografia e Estatística (IBGE). A POF estratificou as famílias em 10 categorias de recebimento mensal, em salários mínimos de setembro de 1996.

As tabelas da POF-1996 identificam 10 categorias de bens de consumo, que foram, Alimentação, Habitação, Vestuário, Transporte, Higiene e Cuidados Pessoais, Assistência à Saúde, Educação, Recreação e Cultura, Fumo e Serviços Pessoais. No presente trabalho, como em Leite Filho (2000), adotou-se apenas 7 . Este procedimento foi adotado devido à necessidade de se adequar às categorias admitidas em Siqueira et al. (1998), Siqueira (1997) e Siqueira (1995) que forneceram as alíquotas efetivas dos impostos indiretos dos diversos bens.
Os $\mathrm{q}_{\mathrm{i}}$ se modificaram porque o governo precisava arrecadar um certo montante de recursos para financiar $\mathrm{F}_{\mathrm{g}}$. Desta maneira, a seguinte restrição orçamentária do governo foi verificada:

$$
\begin{aligned}
& \mathrm{R}+\mathrm{F}_{\mathrm{g}}=\sum \mathrm{t}_{\mathrm{i}} \mathrm{X}_{\mathrm{i}} \\
& \mathrm{F}_{\mathrm{g}}=\mathrm{JI}
\end{aligned}
$$

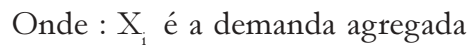
pelo bem i; e $\mathrm{R}$ é a receita arrecadada através dos impostos indiretos para financiar os outros gastos do governo.

Leite Filho (2000) considerou as seguintes propostas de modificações nos impostos indiretos com o objetivo de arrecadar os recursos monetários necessários para financiar o fundo de universalização do acesso à telefonia fixa .

i) Proposta 1: aumento proporcional em todos os impostos indiretos;

ii) Proposta 2: aumento proporcional no Imposto sobre Circulação de Mercadorias e Serviços (ICMS) sobre todos os bens;

iii) Proposta 3: aumento proporcional do ICMS sobre telefone;

iv) Proposta 4: aumento proporcional de um Imposto sobre $\mathrm{Va}$ lor Agregado (IVA) uniforme para todos os bens.

De posse das alíquotas modificadas dos impostos indiretos, encontra-
Tabela 5- Brasil - Preços Modificados dos Bens para Financiar o Acesso ao Sistema Telefônico - Grupo A de Famílias

\begin{tabular}{lrrrr}
\hline Bens & Proposta 1 & Proposta 2 & Proposta 3 & Proposta 4 \\
\hline Alimentação & 1,22579 & 1,22580 & 1,22553 & 1,22020 \\
Habitação & 1,13870 & 1,13875 & 1,13861 & 1,22020 \\
Telefone & 1,41588 & 1,41600 & 1,44920 & 1,22020 \\
Vestuário & 1,27757 & 1,27762 & 1,27310 & 1,22020 \\
Transporte & 1,29555 & 1,29550 & 1,29540 & 1,22020 \\
Cuidados Pessoais & 1,11855 & 1,11858 & 1,11847 & 1,22020 \\
Outros & 1,41988 & 1,42005 & 1,41964 & 1,22020 \\
\hline
\end{tabular}

Fonte : Cálculo realizado pelo autor utilizando a equação 6 juntamente com as propostas.

Tabela 6 - Brasil - Preços Modificados dos Bens para Financiar o Acesso ao Sistema Telefônico - Grupo B de Famílias

\begin{tabular}{lrrrr}
\hline Bens & Proposta 1 & Proposta 2 & Proposta 3 & Proposta 4 \\
\hline Alimentação & 1,22564 & 1,22565 & 1,22553 & 1,22016 \\
Habitação & 1,13867 & 1,38868 & 1,13861 & 1,22016 \\
Telefone & 1,41570 & 1,41576 & 1,42850 & 1,22016 \\
Vestuário & 1,27744 & 1,27746 & 1,27731 & 1,22016 \\
Transporte & 1,29540 & 1,29542 & 1,29527 & 1,22016 \\
Cuidados Pessoais & 1,11853 & 1,11854 & 1,11847 & 1,22016 \\
Outros & 1,41986 & 1,41986 & 1,41964 & 1,22016 \\
\hline
\end{tabular}

Fonte : Cálculo realizado pelo autor utilizando a equação 6 juntamente com as propostas. 
O procedimento de diminuir o número de categorias de bens adotado neste trabalho implicou em fusões em relação às categorias originais da POF-1996. As categorias adotadas aqui foram divididas da seguinte forma, como mostra a tabela 7: Alimentação, Habitação (sem os gastos com telefone), Telefone (obtida a partir do item impostos e taxas, da categoria Habitação), Vestuário, Transporte, Cuidados Pessoais (que englobou as categorias Higiene, Serviços Pessoais e Assistência Médica da POF) e Outros (que englobou Educação, Recreação e Cultura e Fumo).

Tabela 7 - Brasil - Despesa de Consumo Média Mensal Familiar, por Classe de Recebimento Mensal Familiar, Segundo os Tipos de Despesas, com Indicação do Tamanho das Famílias para 11 Regiões Metropolitanas do Brasil (R\$)

\begin{tabular}{|c|c|c|c|c|c|c|c|c|c|c|}
\hline \multirow{2}{*}{$\begin{array}{l}\text { Categoria } \\
\text { de Gastos }\end{array}$} & \multicolumn{10}{|c|}{ Classes de Recebimentos Mensais } \\
\hline & (1) & (2) & (3) & (4) & (5) & (6) & (7) & (8) & (9) & (10) \\
\hline \multicolumn{11}{|l|}{ Despesas de } \\
\hline Consumo & 207.96 & 317.30 & 409.93 & 512.35 & 615.50 & 712.22 & 915.59 & 1266.81 & 1535.91 & 2680.1 \\
\hline Alimentacão & 77.74 & 124.00 & 146.42 & 165.94 & 192.78 & 211.56 & 240.23 & 290.48 & 348.96 & 507.59 \\
\hline Habitação & 53,32 & 80,05 & 106,06 & 140,01 & 165,94 & 192,50 & 255,30 & 354,08 & 431,51 & 808,18 \\
\hline Telefone & 4,37 & 6,76 & 9,24 & 11,84 & 14,53 & 17,6 & 23,07 & 31,76 & 35,89 & 65,08 \\
\hline Vestuário & 11,59 & 20,51 & 27,67 & 36,64 & 44,31 & 52,13 & 68,88 & 92,52 & 102,27 & 185,41 \\
\hline Transporte & 21,31 & 33,61 & 52,24 & 66,3 & 84,23 & 94,96 & 133,27 & 19,29 & 242,26 & 448,93 \\
\hline \multicolumn{11}{|l|}{ Cuidados } \\
\hline Pessoais & 26,25 & 33,69 & 41,40 & 54,61 & 64,33 & 83,76 & 107,46 & 155,66 & 192,93 & 324,57 \\
\hline Outros & 13,38 & 18,68 & 26,90 & 36,99 & 49,38 & 59,63 & 87,38 & 143,02 & 182,09 & 340,45 \\
\hline $\begin{array}{l}\% \text { de } \\
\text { Famílias } \\
\mathrm{N}^{\circ} \text { de }\end{array}$ & 0,098 & 0,081 & 0,14 & 0,065 & 0,102 & 0,071 & 0,122 & 0,069 & 0,068 & 0,107 \\
\hline $\begin{array}{l}\text { Famílias } \\
\text { Tamanho }\end{array}$ & 1324706 & 1116871 & 1918349 & 913755 & 1371262 & 9943384 & 1625967 & 958800 & 950479 & 1369494 \\
\hline Médio & 2,95 & 3,40 & 3,71 & 3,74 & 3,80 & 3,90 & 3,87 & 3,93 & 3,78 & 3,67 \\
\hline
\end{tabular}

Fonte: IBGE - POF 1995-96.

O presente estudo, como em Leite Filho (2000), utilizou para as funções utilidade indireta e demanda das unidades familiares o Sistema de Despesa Linear (SDL). Para fins de comparação utilizou-se também a especificação Cobb-Douglas para aquelas funções. A função utilidade indireta e a função demanda para o SDL têm as seguintes formas, respectivamente:

$$
\begin{array}{cc}
\mathrm{u}^{\mathrm{j}}=\left(\mathrm{Y}^{\mathrm{j}}-\sum_{\mathrm{i}=1}^{\mathrm{n}} \mathrm{q}_{\mathrm{i}} \alpha_{\mathrm{i}}\right) / \prod_{\mathrm{i}} \mathrm{q}_{\mathrm{i}}^{\beta \mathrm{i}} & \mathrm{i}=1, \ldots, \mathrm{n} \quad(9) \\
\mathrm{q}_{\mathrm{i}} \mathrm{x}_{\mathrm{i}}=\mathrm{q}_{\mathrm{i}} \alpha_{\mathrm{i}}+\beta_{\mathrm{i}}\left(\mathrm{Y}^{\mathrm{j}}-\sum_{\mathrm{i}}^{\mathrm{n}} \mathrm{q}_{\mathrm{i}} \alpha_{\mathrm{i}}\right) & \mathrm{i}=1, \ldots, \mathrm{n} \quad(10)
\end{array}
$$

Onde: $\Sigma \beta i=1 ; \beta i>0$ e xi $-\alpha i>0$. Os $\alpha$ i e $\beta$ i são os parâmetros comportamentais. $\mathrm{O} \alpha$ i é parâmetro referente aos gastos de subsistência no bem $\mathrm{i}$, enquanto $\beta \mathrm{i}$ indica o percentual da renda supranumerária que é gasta no bem $i$.

$\mathrm{Na}$ forma funcional Cobb-Douglas todos os $\alpha$ i são iguais a zero. Portanto, temos que as funções utilidade indireta e demanda têm as seguintes formas:

$$
\begin{gathered}
\mathrm{u}^{\mathrm{j}}=\mathrm{Y}_{\mathrm{i}}^{\mathrm{j} /} \prod_{\mathrm{i}}{ }^{\lambda \mathrm{i}} \\
\mathrm{X}_{\mathrm{i}} \mathrm{q}_{\mathrm{i}}=\lambda_{\mathrm{i}} \mathrm{Y}^{\mathrm{j}}
\end{gathered}
$$

Onde l é a parcela orçamentária média, que representa os gastos médios das famílias, em valores percentuais, com os diversos bens. Os valores dos parâmetros comportamentais são:

Tabela 8 - Brasil - Parâmetros $\beta$ do Sistema de Despesa Linear

\begin{tabular}{lcccc}
\hline Categorias de Gastos & $\beta_{\mathbf{i}}$ & $\sigma$ & $\mathbf{t}$ & $\mathbf{R}^{2}$ \\
\hline Alimentação & 0,23338 & 0,59813 & 3,901818 & 0,92259 \\
Habitação & 0,296805 & 0,023075 & 12,86285 & 0,961073 \\
Telefone & 0,2752 & 0,003221 & 8,544262 & 0,915711 \\
Vestuário & 0,8596 & 0,01216 & 7,133609 & 0,883813 \\
Transporte & 0,161234 & 0,061421 & 2,625067 & 0,497517 \\
Cuidados Pessoais & 0,091063 & 0,02678 & 3,400343 & 0,631432 \\
Outros & 0,104038 & 0,030062 & 3,460826 & 0,840233 \\
$\Sigma$ & 1 & & & \\
\hline
\end{tabular}

Fonte : Cálculo realizado pelo autor utilizando o programa eviews.

o parâmetro comportamental a foi calculado com base na hipótese de que os gastos de subsistência assumem o valor de $\mathrm{R} \$ 30,27$, que foi obtido dividindo o salário mínimo prevalecente em 1995-96, R $\$ 112,00$, pelo tamanho médio de todas as unidades familiares, 3,7 admitida na POF-1996. Nas tabelas 9 e 10 estão os valores de a para as regiões metropolitanas e não metropolitanas, segundo as hipóteses acima. Posteriormente a tabela 12 exibe os valores do parâmetro comportamental para a especificação Cobb-Douglas. Este foi obtido dividindo os gastos em cada categoria pelo gasto total, encontrando o gasto médio.

Tabela 9 - Brasil - Parâmetros $\alpha$ do Sistema de Despesa Linear para as 10 classes de Despesa de Consumo da POF1995-96 para as Regiões Metropolitanas, Alternativa (b)

\begin{tabular}{lccccccccccc}
\hline $\begin{array}{c}\text { Categoria } \\
\text { de Gastos }\end{array}$ & \multicolumn{10}{c}{ Categoria de Unidades Familiares } \\
\cline { 2 - 12 } & $\mathbf{( 1 )}$ & $\mathbf{( 2 )}$ & $\mathbf{( 3 )}$ & $\mathbf{( 4 )}$ & $\mathbf{( 5 )}$ & $\mathbf{( 6 )}$ & $\mathbf{( 7 )}$ & $\mathbf{( 8 )}$ & $\mathbf{( 9 )}$ & $\mathbf{( 1 0 )}$ \\
\hline Alimentação & 40,4 & 60,35 & 62,79 & 59,39 & 61,99 & 59,48 & 44,32 & 17,57 & 14,07 & $-75,06$ \\
Habitação & 15,90 & 14,42 & 15,56 & 18,92 & 15,27 & 14,18 & 16,57 & 10,58 & 8,53 & 40,09 \\
Telefone & 0,76 & 0,60 & 0,74 & 0,60 & 0,54 & 0,93 & 0,81 & 0,03 & $-2,33$ & $-3,97$ \\
Vestuário & 1,09 & 1,63 & 1,63 & 1,82 & 1,00 & 0,82 & 0,31 & $-5,12$ & $-15,62$ & $-27,73$ \\
Transporte & 1,68 & $-0,73$ & 3,28 & 1,51 & 2,73 & $-0,64$ & 3,73 & 10,41 & 10,09 & 26,79 \\
Cuidados & & & & & & & & & & \\
Pessoais & 13,81 & 12,66 & 12,78 & 16,33 & 16,76 & 26,51 & 31,22 & 45,35 & 59,77 & 81,02 \\
Outros & 0,73 & $-2,55$ & $-2,86$ & $-3,19$ & $-1,89$ & $-1,54$ & 3,14 & 16,29 & 24,05 & 51,53 \\
\hline
\end{tabular}

Fonte : Cálculo realizado pelo autor utilizando a hipótese de que os gastos de subsistência assumem o valor de $\mathbf{R} \$ 30,27$, que foi obtido dividindo o salário mínimo prevalecente em 1995-96, $R \$ 112,00$, pelo tamanho

\begin{tabular}{|c|c|c|c|c|c|c|c|c|c|c|}
\hline \multirow{2}{*}{$\begin{array}{l}\text { Categoria } \\
\text { de Gastos }\end{array}$} & \multicolumn{10}{|c|}{ Categoria de Unidades Familiares } \\
\hline & (1) & (2) & (3) & (4) & (5) & (6) & (7) & (8) & (9) & (10) \\
\hline Alimentação & 20,42 & 3,17 & 31,39 & 29,69 & 30,99 & 29,74 & 22,16 & 8,78 & 7,03 & $-37,53$ \\
\hline Habitação & 7,95 & 7,21 & 7,78 & 9,46 & 7,63 & 7,09 & 8,28 & 5,29 & 4,26 & 20,04 \\
\hline Telefone & 0,38 & 0,30 & 0,37 & 0,30 & 0,27 & 0,46 & 0,40 & 0,01 & $-1,16$ & $-1,98$ \\
\hline Vestuário & 0,54 & 0,81 & 0,81 & 0,91 & 0,50 & 0,41 & 0,15 & $-2,56$ & $-7,81$ & $-13,86$ \\
\hline Transporte & 0,84 & $-0,36$ & 1,64 & 0,75 & 1,36 & $-0,32$ & 1,86 & 5,20 & 5,04 & 13,39 \\
\hline Cuidac & & & & & & & & & & \\
\hline & 6,90 & 6,33 & 6,39 & 8,16 & 8,38 & 13,25 & 15,61 & 22,67 & 28,38 & 40,51 \\
\hline Outros & 0,36 & $-1,27$ & $-1,43$ & $-1,59$ & $-0,94$ & $-0,77$ & 1,57 & 8,14 & 12,02 & 25,76 \\
\hline
\end{tabular}
médio de todas as unidades familiares, 3,7 .

Tabela 10 - Brasil - Parâmetros $\alpha$ do Sistema de Despesa Linear para as 10 classes de Despesa de Consumo da POF1995-96 para as Regiões Não Metropolitanas, Alternativa (b)

Fonte : Cálculo realizado pelo autor utilizando a hipótese de que os gastos de subsistência assumem o valor de $R \$ 30,27$, que foi obtido dividindo o salário mínimo prevalecente em 1995-96, $R \$ 112,00$, pelo tamanho médio de todas as unidades familiares, 3,7. 
Tabela 11 - Brasil - Parâmetros 1 do Sistema Cobb-Douglas para as 10 Classes de Despesa de Consumo da POF- 1995-96.

\begin{tabular}{lccccccccccc}
\hline $\begin{array}{l}\text { Categoria } \\
\text { de Gastos }\end{array}$ & \multicolumn{10}{c}{ Categoria de Unidades Familiares } \\
\cline { 2 - 12 } & $(1)$ & $(2)$ & $(3)$ & $(4)$ & $(5)$ & $(6)$ & $(7)$ & $(8)$ & $(9)$ & $(10)$ \\
\hline Alimentação & 0,374 & 0,391 & 0,357 & 0,324 & 0,313 & 0,297 & 0,262 & 0,229 & 0,27 & 0,189 \\
Habitação & 0,256 & 0,252 & 0,259 & 0,273 & 0,270 & 0,270 & 0,79 & 0,280 & 0,281 & 0,302 \\
Telefone & 0,021 & 0,021 & 0,023 & 0,023 & 0,024 & 0,025 & 0,025 & 0,025 & 0,023 & 0,024 \\
Vestuário & 0,056 & 0,065 & 0,067 & 0,072 & 0,072 & 0,073 & 0,075 & 0,073 & 0,067 & 0,069 \\
Transporte & 0,102 & 0,106 & 0,127 & 0,129 & 0,137 & 0,133 & 0,146 & 0,157 & 0,158 & 0,167 \\
Cuidados & & & & & & & & & & \\
Pessoais & 0,126 & 0,106 & 0,101 & 0,107 & 0,105 & 0,118 & 0,117 & 0,123 & 0,126 & 0,121 \\
Outros & 0,064 & 0,059 & 0,066 & 0,072 & 0.080 & 0.084 & 0,095 & 0,113 & 0,119 & 0,127 \\
\hline
\end{tabular}

Fonte : Cálculo realizado pelo autor e foi obtido dividindo os gastos em cada categoria pelo gasto total.

\section{ESPECIFICAÇÃO DA VARIAÇÃO EQUIVALENTE}

Em seção anterior mostramos que a variação equivalente (VE) é a medida adotada neste estudo para avaliar os impactos que o financiamento público à universalização do acesso à telefonia fixa no Brasil têm sobre o bem-estar das famílias. Deste modo, a VE pode ser aceita como uma indicação dos possíveis benefícios obtidos pelas famílias por causa da universalização do acesso àquele sistema.

A VE, como foi visto anteriormente, é baseada na função utilidade de métrica monetária que, por sua vez, é uma transformação monotônica da função gasto. Desta forma, para obtermos a VE temos que primeiramente dispor desta última função. Para o sistema de demanda linear (SDL), a função gasto tem a seguinte forma:

$$
\mathrm{e}=\mathrm{u}_{\mathrm{i}}^{\mathrm{j}} \prod_{\mathrm{i}} \mathrm{q}^{\beta \mathrm{i}}+\sum \mathrm{q}_{\mathrm{i}} \alpha_{\mathrm{i}}
$$

Para o sistema Cobb-Douglas, tem-se:

$$
\mathrm{e}=\mathrm{u}^{\mathrm{j}} \Pi \mathrm{q}_{\mathrm{i}}^{\lambda_{\mathrm{i}}}
$$

A VE para o SDL e o sistema Cobb-Douglas são, respectivamente $^{10}$ :

$$
V E=\left(Y_{j}^{1}-\sum_{i} q_{i}^{1} \alpha_{i}\right) \prod_{i}\left(q_{i}^{0} / q_{i}^{1}\right)^{\beta i}-Y_{j}^{0}+\sum_{i} q_{i}^{0} \alpha_{i}
$$

e

$$
V E=Y_{j}^{1} \prod_{i}\left(q_{i}^{0} / q_{i}^{1}\right)^{\lambda i}-Y_{j}^{0}
$$

Onde : $\mathrm{Y}^{0}=\mathrm{A}$ renda da família $\mathrm{j}$ antes das modificações nos impostos indiretos;

$\mathrm{Y}^{1}{ }_{\mathrm{j}}=\mathrm{A}$ renda da família $\mathrm{j}$ depois das modificações nos impostos indiretos;

$\mathrm{q}_{\mathrm{i}}^{0}=$ preço do bem $\mathrm{i}$ antes das modificações dos impostos indiretos;

$\mathrm{q}_{\mathrm{i}}^{1}=$ preço do bem $\mathrm{i}$ depois das modificações dos impostos indiretos;

$\alpha_{i}, \beta_{i}$ e $\lambda_{i}=$ parâmetros comportamentais do SDL e do sistema Cobb-Douglas.

\section{OS RESULTADOS}

Os valores contidos nas tabelas mostram que, com os dados disponíveis e o método adotado, o impacto percentual mensal sobre o bem-estar de todas as famílias é positivo, ou seja, os benefícios da universalização do acesso são maiores do que os custos de implementá-la. Este resultado é análogo ao encontrado em Leite Filho (2000). Desta feita, os resultado daquele estudo, bem como deste, sugerem que a universalização do acesso pode ser vista como uma melhoria de Pareto, dado que todas as famílias se encontram numa situação melhor e nenhuma delas pior.

O resultado geral descrito acima pode ser explicado por três motivos. Primeiro, o fundo necessário para financiar a universalização, obtido com as modificações dos impostos indiretos, foi, em termos percentuais, muito pequeno em relação ao montante arrecadado para o ano de $1996^{11}$ e, desta forma, o custo da universalização para as famílias foi pequeno em termos percentuais.

Tabela 12 - Brasil - Variação Equivalente(VE) para Sistema de Demanda Linear- Todas as Propostas de Modificação dos Impostos Indiretos para Financiar o grupo A de Famílias Região Metropolitana ( $\%$ da renda mensal)

\begin{tabular}{ccccc}
\hline $\begin{array}{l}\text { Classes de } \\
\text { Recebimento Mensal }\end{array}$ & VE1 & VE2 & VE3 & VE4 \\
\hline 1 & 0,667 & 0,666 & 0,665 & 0,651 \\
2 & 0,437 & 0,436 & 0,435 & 0,426 \\
3 & 0,339 & 0,338 & 0,337 & 0,334 \\
4 & 0,271 & 0,270 & 0,269 & 0,266 \\
5 & 0,226 & 0,225 & 0,224 & 0,227 \\
6 & 0,195 & 0,194 & 0,193 & 0,194 \\
7 & 0,152 & 0,151 & 0,150 & 0,156 \\
8 & 0,109 & 0,108 & 0,107 & 0,121 \\
9 & 0,091 & 0,090 & 0,089 & 0,101 \\
10 & 0,052 & 0,051 & 0,051 & 0,065 \\
\hline
\end{tabular}

Fonte: Resultados obtidos pelo autor utilizando o programa GAMS. obs. VE1 - variação equivalente para proposta 1, VE2variação equivalente para proposta 2 , VE3- variação e quivalente para proposta 3, VE4- variação equivalente para proposta 4.

Tabela 13 - Brasil - Variação Equivalente(VE) para Sistema de Demanda Linear - Todas as Propostas de Modificação dos Impostos Indiretos para Financiar o grupo A de FamíliasRegião Não Metropolitana (\% da renda mensal)

\begin{tabular}{ccccc}
\hline $\begin{array}{l}\text { Classes de } \\
\text { Recebimento Mensal }\end{array}$ & VE1 & VE2 & VE3 & VE4 \\
\hline 1 & 1,336 & 1,335 & 1,334 & 1,322 \\
2 & 0,876 & 0,875 & 0,874 & 0,867 \\
3 & 0,678 & 0,667 & 0,676 & 0,675 \\
4 & 0,542 & 0,541 & 0,541 & 0,539 \\
5 & 0,451 & 0,450 & 0,450 & 0,453 \\
6 & 0,390 & 0,389 & 0,385 & 0,391 \\
7 & 0,303 & 0,302 & 0,302 & 0,308 \\
8 & 0,219 & 0,218 & 0,218 & 0,231 \\
9 & 0,181 & 0,180 & 0,180 & 0,193 \\
10 & 0,104 & 0,103 & 0,102 & 0,117 \\
\hline
\end{tabular}

Obs.: A fonte é a mesma daquele daTabela 12.

10 Creedy (1997). Creedy E Martin (1997), Gabel e Kennett (1993), Pollak e Wales (1978) e Rosen (1976).

11 Ver Siqueira et al. (1998). 
Terceiro, não foi considerado os efeitos cruzados na demanda dos bens decorrentes das modificações nas alíquotas dos impostos indiretos, dado que o método adotado para mensurar os impactos sobre o bem-estar das famílias não utilizou um modelo de equilíbrio geral e, sim, de equilíbrio parcial. Uma análise de eficiência e distribuição mais exata deveria levar em consideração este fato. Sendo assim, o resultado geral pode ser interpretado como uma aproximação do verdadeiro.

Tabela 15 - Brasil - Variação Equivalente(VE) para Sistema de Demanda Linear - Todas as Propostas de Modificação dos Impostos Indiretos para Financiar o grupo B de Famílias - Região Não Metropolitana (\% da renda mensal)

\begin{tabular}{ccccc}
\hline $\begin{array}{l}\text { Classes de } \\
\text { Recebimento Mensal }\end{array}$ & VE1 & VE2 & VE3 & VE4 \\
\hline 1 & 1,335 & 1,334 & 1,333 & 1,322 \\
2 & 0,875 & 0,874 & 0,873 & 0,867 \\
3 & 0,667 & 0,666 & 0.675 & 0,675 \\
4 & 0,542 & 0,541 & 0,540 & 0,539 \\
5 & 0,451 & 0,450 & 0,449 & 0,454 \\
6 & 0,390 & 0,389 & 0,388 & 0,418 \\
7 & 0,304 & 0,303 & 0,301 & 0,309 \\
8 & 0,219 & 0,218 & 0,217 & 0,231 \\
9 & 0,181 & 0,180 & 0,179 & 0,192 \\
10 & 0,104 & 0,103 & 0,102 & 0,118 \\
\hline
\end{tabular}

Obs.: A fonte é a mesma daquela daTabela 12.

Mesmo apresentado àquelas deficiências o trabalho tem a importância de mostrar que a universalização do acesso ao sistema telefônico fixo, embora aproximadamente, exibiu impactos positivos no bem-estar das famílias e, por conta disso, pôde se constituir em política governamental.

Analisando mais atentamente as tabelas expostas anteriormente observa-se que os impactos positivos foram maiores para aquelas famílias de ganhos mensais mais baixos. $\mathrm{O}$ impacto positivo mensal ficou em torno de $0,667 \%$ para as famílias das regiões metropolitanas e de 1,336\% para aquelas das regiões não metropolitanas.

As famílias de ganhos mensais mais altos também tiveram impacto positivo, dado que a VE apresenta valor positivo, como mostram as tabelas. Os valores da VE para essas classes de recebimento foram menores em termos percentuais porque o percentual de gasto para estas famílias em bens nos quais os impostos indiretos incidiram de forma mais acentuada devem ser maiores do que os verificados para as famílias menos abastadas.

Em termos dos impactos nas duas regiões, aquelas famílias situadas nas regiões não metropolitanas tiveram um impacto maior em seu bem-estar, representado por uma VE de maior valor, em relação àquele das regiões metropolitanas.

A diferença no impacto sobre o bem-estar das famílias entre o financiamento para os grupos de famílias A e B é mínima, como se percebe pela comparação dos resultados das simulações contidas nas tabelas 12, 13 e 16 com aqueles das tabelas 14,15 e 17.
O número de famílias do grupo de famílias A é quase o dobro do B, como mostra a tabela 2 e, mesmo assim, as diferenças nos impactos no bem-estar das famílias são muito pequeno. No tocante às propostas de modificações dos impostos indiretos, a que permitiu maior ganho percentual, em geral, foi aquela que aumenta proporcionalmente todos os impostos indiretos, muito embora as diferenças nos impactos sobre o bem-estar se mostrem extremamente pequenas entre as quatro propostas.

Um fato que deve ser ressaltado é que a partir da $5^{\mathrm{a}}$ classe de ganhos mensais, o aumento proporcional do Imposto sobre Valor Agregado (IVA) uniforme para todos os bens gera um impacto positivo maior, em termos percentuais, no bem-estar das famílias de ambas as regiões do que todas as outras três propostas anteriores. Os resultados para as classes de recebimentos mensais anteriores à $5^{\mathrm{a}}$ seguem a tendência de que a primeira proposta gera um impacto positivo maior.

Este resultado se justifica pelo fato de que a proposta quatro acarreta uma redução nos gastos das classes de ganhos mais elevados, dado que os preços dos bens a que estas dedicam maior parcela de sua renda diminuíram com a fixação de um imposto sobre valor agregado (IVA) uniforme para todos os bens.

Tabela 16 - Brasil - Variação Equivalente(VE) para Sistema de Demanda Cobb-Douglas - Todas as Propostas de Modificação dos Impostos Indiretos para Financiar o grupo A de Famílias - Região Não Metropolitana ( $\%$ da renda mensal)

\begin{tabular}{ccccc}
\hline $\begin{array}{l}\text { Classes de } \\
\text { Recebimento Mensal }\end{array}$ & VE1 & VE2 & VE3 & VE4 \\
\hline 1 & 1,336 & 1,335 & 1,335 & 1,313 \\
2 & 0,875 & 0,875 & 0,874 & 0,862 \\
3 & 0,677 & 0,677 & 0,676 & 0,674 \\
4 & 0,542 & 0,541 & 0,541 & 0,537 \\
5 & 0,451 & 0,451 & 0,450 & 0,457 \\
6 & 0,390 & 0,389 & 0,389 & 0,390 \\
7 & 0,303 & 0,303 & 0,302 & 0,309 \\
8 & 0,219 & 0,219 & 0,218 & 0,232 \\
9 & 0,181 & 0,181 & 0,180 & 0,194 \\
10 & 0,103 & 0,103 & 0,103 & 0,119 \\
\hline
\end{tabular}

Obs.: A fonte é a mesma daquela daTabela 12 .

De uma forma geral, os resultados indicam que, em qualquer uma das propostas de modificações dos impostos indiretos que for adotada, há impactos positivos para todas as classes de recebimento mensal. Ou seja, as modificações nos impostos indiretos não compensaram a concessão do montante monetário para que as famílias adquiram o acesso à telefonia fixa, o que implica em aumento de renda para as mesmas e, por sua vez, em seu bem-estar.

Os valores contidos nas tabelas 16, 17 e 18 foram obtidos utilizando a especificação Cobb-Douglas para a VE. Esses resultados revelam que não existe praticamente diferença em comparação com aqueles obtidos utilizando SDL. Desta forma, o estudo conclui que, independentemente da formulação adotada para a função gasto e variação equivalente, do ponto de vista das famílias a universalização do acesso ao sis- 
tema de telecomunicação fixo aumenta o bem-estar destas porque pode proporcionar um aumento hipotético de renda, dado que as transferências lump sum para que as mesmas adquiram acesso aquele sistema é maior que as modificações ocorridas nos impostos indiretos. Este resultado, mais uma vez, corrobora o encontrado em Leite Filho (2000).

Tabela 17 - Brasil - Variação Equivalente(VE) para Sistema de Demanda Cobb-Douglas - Todas as Propostas de Modificação dos Impostos Indiretos para Financiar o grupo B de Famílias - Região Metropolitana ( $\%$ da renda mensal)

\begin{tabular}{ccccc}
\hline $\begin{array}{c}\text { Classes de } \\
\text { Recebimento Mensal }\end{array}$ & VE1 & VE2 & VE3 & VE4 \\
\hline 1 & 0,667 & 0,667 & 0,666 & 0,648 \\
2 & 0,437 & 0,437 & 0,436 & 0,426 \\
3 & 0,338 & 0,338 & 0,337 & 0,335 \\
4 & 0,271 & 0,270 & 0,269 & 0,266 \\
5 & 0,225 & 0,255 & 0,223 & 0,228 \\
6 & 0,194 & 0,194 & 0,193 & 0,195 \\
7 & 0,151 & 0,151 & 0,149 & 0,157 \\
8 & 0,109 & 0,109 & 0,108 & 0,122 \\
9 & 0,009 & 0,089 & 0,088 & 0,103 \\
10 & 0,051 & 0,051 & 0,050 & 0,066 \\
\hline
\end{tabular}

Obs.: A fonte é a mesma daquela da'Tabela 12.

Tabela 18 - Brasil - Variação Equivalente(VE) para Sistema de Demanda Cobb-Douglas - Todas as Propostas de Modificação dos de Impostos Indiretos para Financiar o grupo B de Famílias - Região Não Metropolitana (\% da renda mensal)

\begin{tabular}{ccccc}
\hline $\begin{array}{l}\text { Classes de } \\
\text { Recebimento Mensal }\end{array}$ & VE1 & VE2 & VE3 & VE3 \\
\hline 1 & 1,335 & 1,335 & 1,333 & 1,312 \\
2 & 0,874 & 0,874 & 0,873 & 0,862 \\
3 & 0,677 & 0,677 & 0,675 & 0,674 \\
4 & 0,541 & 0,541 & 0,540 & 0,537 \\
5 & 0,451 & 0,451 & 0,449 & 0,453 \\
6 & 0,389 & 0,389 & 0,388 & 0,391 \\
7 & 0,302 & 0,302 & 0,301 & 0,309 \\
8 & 0,219 & 0,218 & 0,217 & 0,232 \\
9 & 0,180 & 0,180 & 0,179 & 0,194 \\
10 & 0,103 & 0,103 & 0,102 & 0,119 \\
\hline
\end{tabular}

Obs.: A fonte é a mesma daquela daTabela 12.

Aqui deve ser novamente ressaltado que os valores da VE obtidos pelas simulações apenas indicam que a universalização do acesso pode gerar benefícios positivos. Portanto, com a VE apresentando valores positivos podemos concluir que a universalização do acesso à telefonia fixa pode constituir-se num dos muitos instrumentos utilizados pelo governo para aumentar o bem-estar das famílias.

\section{CONCLUSÃO}

Como foi dito anteriormente, o objetivo estabelecido para este trabalho foi o de avaliar os impactos sobre o bemestar das famílias brasileiras decorrentes do aumento do número de usuários do sistema de telefonia fixa. O mesmo sur- giu da observação dos resultados exibidos em Leite Filho (2000). Aqueles foram que o impacto do aumento na quantidade de novos usuários no bem-estar da sociedade brasileira era positivo, tanto para o quantitativo estabelecido pela ANATEL como para o alternativo estabelecido com base na renda dos não usuários. Ou seja, o benefício de ter o acesso ao sistema de telefonia fixa é maior do que os custos de viabilizá-lo. Naquele contexto, os aumentos dos impostos indiretos foram compensados pelo benefício do acesso.

Mas, como comentado antes, a sociedade brasileira é composta de famílias com perfis de renda/gastos distintos e que podem exibir impactos diferenciados no seu bem-estar quando os impostos indiretos sofrem elevação para viabilizar o acesso à telefonia fixa. Ou seja, para algumas famílias o benefício do acesso à telefonia fixa poderia ser suplantado pelo custo representado pelo aumento dos impostos indiretos, mesmo que para a sociedade os benefícios suplantem os custos.

Como vimos na seção anterior, aquele resultado obtido em Leite Filho (2000) foi confirmado para todas as classes de renda da sociedade brasileira, com os dados da POF de 1996. Ou seja, o bem-estar das famílias brasileiras sofreria um impacto positivo se o governo brasileiro fizesse a universalização do acesso à telefonia fixa.

Sendo assim, pode-se afirmar que, com os dados da POF de 1996, a universalização do acesso à telefonia fixa é uma melhoria de Pareto, dado que beneficiou todos sem prejudicar ninguém. Com base neste resultado, e os exibidos em Leite Filho (2000), pode-se concluir que, em termos de políticas públicas, a decisão de universalizar o acesso à telefonia seria de bom tom para a sociedade brasileira.

\section{REFERÊNCIAS BIBLIOGRÁFICAS}

CREEDY, J. Measuring the Welfare Effects of Price Changes: A Convenient Parametric Approach., Institute Working Paper, Melbourne, 1997. n. 1/97, p. $1-24$.

CREEDY, J.; MARTIN, C. Estimative of the Linear Expenditure System Using the Household Expenditure Survey. Institute Working Paper, Melbourne, 1997. n. 12/97, p. 1-15.

ECONOMIDES, N. ; WHITE, L.J. Networks and Compatibility: Implications for Antitrust. European Economic Review. 1994. v. 38, p. 651-662.

ECONOMIDES, N. The Economics of Networks. International Journal of Industrial Organization. 1996. v. 42 , p. 352-380

GABEL, D. ; KENNETT, E. Pricing of Telecomunications Services. Review of Industrial Organization, 1993. v. 8, n. 4, p. 797-801

GRAHAM, S.; CORNFORD, J.; MARVIN, S. The Socio-Economic Benefits of a Universal Telephone Network: A Demand-Side View of Universal Service. Telecomunication Policy. 1998. v. 20, n. 1, p. 3-10.

IBGE. Pesquisa de Orçamento Familiar 1995-1996. Rio de Janeiro: IBGE. 1997. IBGE. 1992.
Matriz de Insumo-Produto: Brasil 1995. Rio de Janeiro: 
Pesquisa Nacional de Amostragem por Domicílio. IBGE. 1996.

KREPS, D. M. A Course in Microeconomic Theory. Cambridge. Harvester Wheatsheaf Press. 1990. 850 p.

LEITE FILHO, P. A. M. Acesso Universal ao Sistema de Telefonia Fixa no Brasil: Esquemas Alternativos de Financiamento e Avaliação dos Impactos Distributivos Sobre o Bem-Estar da Sociedade. CAMPINAS/SP. Anais do Congresso da ANPEC. DEZ .2000.

MAS-COLLEL, A. et al. Microeconomic Theory. Oxford: Oxford University Press. 1995. 981 p.

NOGUEIRA, J.R. ET AL Financiamento do Acesso Universal às Telecomunicações no Brasil: Análise de Esquemas Tributários Alternativos. Relatório Final de Pesquisa. UFPE. Recife - PE. 1998.

POLLAK, R A. and WALES, T. J. Estimation of complete demand systems from household budget data: the linear and quadratic expenditure systems. American Economic Review, 1978. v. 68 n. 3 p.348-359.
ROSEN, H. S. A Methodology for Evaluating Tax Reform Proposals. Journal of Public Economics. 1976. v. 6 n. 6 p. 105-121.

SIQUEIRA, R. B. Redistributive Indirect Taxes for Brazil: An Application of the Theory of Optimal Taxation. Tese Doutoral. Londres: University of London. 1995. 115 p.

Redistributive Effects of Alternative Indirect Tax Reforms for Brazil, Revista de Economia Aplicada. Rio de Janeiro, v. 1, n. 3, p. 349-372. 1997. SIQUEIRA, R. B. et al. Uma Análise da Incidência Final dos Impostos Indiretos No Brasil. Recife, Departamento de Economia da UFPE . 1998.mimeo.

TAYLOR, L. D. Telecomunication Demand in Theory and Pratice. New York: Kluwer Academic Publishers, 1994. 385 p.

VARIAN, H.R. Microeconomic Analysis, 3. ed.. New York: W. W. Norton, 1992. $506 \mathrm{p}$. 